
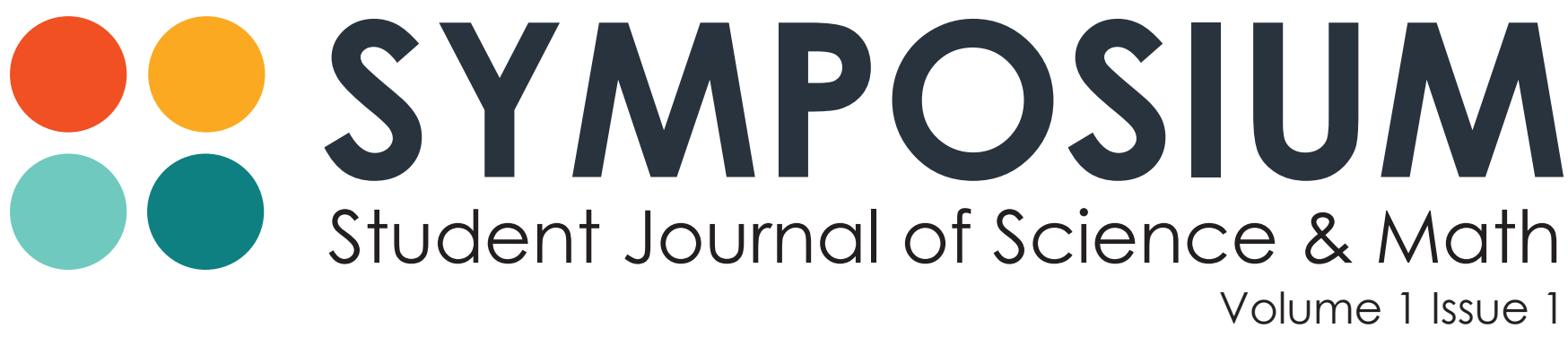


\title{
VISCOSITY DEPENDENCE OF FARADAY WAVE FORMATION THRESHOLDS
}

\author{
A RESEARCH ARTICLE
}

by Lisa M. Slaughter

\begin{abstract}
This experiment uses an electromagnetic shaker to produce standing wave patterns on the surface of a vertically oscillating sample of silicon liquid. These surface waves, known as Faraday waves, form shapes such as squares, lines, and hexagons. They are known to be dependent upon the frequency and amplitude of the forcing as well as on the viscosity and depth of the liquid in the dish. At a depth of $4 \mathrm{~mm}$ and for various silicon liquids having kinematic viscosities of $\mathrm{Io}, 20$, and $38 \mathrm{cSt}$, we determined the acceleration at which patterns form for frequencies between Io and $60 \mathrm{~Hz}$. For the Io and $20 \mathrm{cSt}$ cases, it was found that the acceleration at which these patterns form does not heavily depend upon the viscosity of their host liquid. This is in stark contrast with the current body of knowledge. The case for $38 \mathrm{cSt}$, however, varies from the other two cases in both value and shape.
\end{abstract}




\section{Introduction}

If you have ever made a wine glass to "sing" and noticed ripples on the surface of the liquid within, you have experienced the phenomenon of Faraday waves. Named for Michael Faraday, who first studied them in I83I (Benjamin \& Purcell, 1954), these standing waves can form into intricate patterns made up of three primary shapes: stripes, squares, and hexagons. They are known to be dependent upon the frequency and amplitude of the forcing waveform, as well as on the viscosity and depth of the host liquid (Kudrolli \& Gollub, 1996). This experiment aims to contribute to the body of knowledge on these waves by investigating the dependence of their formation threshold upon the viscosity of their host liquid. Using an electromagnetic shaker, patterns are produced on the surface of a vertically oscillating sample of silicon liquid. The viscosity of this liquid is varied, and the threshold of pattern formation is measured as a function of the frequency and maximum acceleration of the forcing.

\section{Theory}

The primary constituents of the patterns formed by Faraday waves are stripes, squares, and hexagons. Stripes occur when a single, continuous wave travels across the surface of the liquid, forming long crests, similar to ocean waves along the shore. These waves have associated with them a mathematical construct, called a k-vector, which describes the waves' direction of propagation. Squares, shown in Figure $\mathrm{I}$, are formed when the k-vectors of two waves cross perpendicularly to each other, and hexagons occur when the k-vectors of three waves cross at $120^{\circ}$ angles of one another. An example of hexagons is shown in Figure 2. In some cases, the patterns formed consist purely of one of these shapes, though usually combinations form, as shown in Figures 3 and 4. These combinations are chaotic and timedependent, causing, for example, a square to appear where once there was a hexagon. The shapes are in competition for the same space, and they appear to melt into each other.

\section{Experimental Apparatus}

A block diagram of the experimental apparatus used in this experiment is shown in Figure 5. An electromagnetic shaker was used to vertically oscillate a dish containing silicon oil of known viscosity at a depth of $4 \mathrm{~mm}$. Proper operation of the shaker requires that a load of 2-5 pounds be applied. Since our sample and the dish containing it weigh only a few grams, a symmetrical aluminum block was machined and fastened to the plate of the shaker to achieve the needed load. To ensure that the oscillations were purely one-dimensional, the load was balanced through an optical method. Two horizontally aligned lasers were reflected off opposing sides of the shaker surface onto a wall. The shaker was then sinusoidally driven, and the motion of the laser points observed. If the laser point motion was not identical, the balance of the plate and shaker was adjusted until the motion of the laser points coincided.

The motion of the shaker was defined using a signal generator. Since the signal generator does not provide enough power to drive the shaker on its own, its output was fed through an intermediary power amplifier before reaching the shaker. An investigation of the shaker's response to changes in 
amplitude was performed, and it was found that use of the amplifier's amplitude control knob resulted in a non-linear amplitude response in the shaker. In order to achieve the desired linear response, it was necessary to maintain static amplifier settings for all measurements and instead use the signal generator controls to alter the original waveform amplitude.

The amplitude of the waveform is defined in terms of voltage by the signal generator. For these experiments, however, the physical amplitude of motion must be known in order to calculate acceleration. To obtain this measurement, a micrometer was used. The zero mark on the micrometer was calibrated to correspond to the upper edge of the dish in equilibrium position. Then, while the signal was applied, the micrometer arm was lowered to the point that it could be heard barely tapping against the dish edge. The number displayed was the amplitude of the upper half of the sinusoidal forcing oscillation. The micrometer and shaker were both fastened to a non-ferrous optical bench to prevent relative motion between them as well as to provide general stability and vibration isolation. It was important that no ferrous materials be used near the shaker, as the sinusoidal nature of its motion could be affected.

A video camera was used to image the patterns forming on the liquid surface. Placed directly above the dish, its line of sight fell perpendicular to the surface of the liquid, ensuring that no parallax occurred. Videos were acquired by data acquisition software that offers a feature where distance measurements can be made against a user-defined scale. To this end, a ruler was placed next to the shaker, so that such a scale is provided in each video.

To properly image the surface waves, they must be lit by bright, uniform lighting from directly above. Since the camera must also be mounted directly above the sample, a custom lighting system was constructed in which eighteen light-emitting diodes are arranged on a double-layer balsawood ring, through which the camera could be aimed. These LEDs are incredibly bright and provide very uniform illumination. The higher the ring is placed above the system, the more uniform the lighting, and the less apparent the reflection of the LEDs is off of the liquid's surface. For this experiment, the ring and camera were placed approximately two feet above the test surface.

The samples under investigation are silicon oils, with manufacturer determined kinematic viscosities of IO, 20, and $38 \mathrm{cSt}$. For reference, water has a kinematic viscosity of I cSt. The depths of these liquids were maintained at $4 \mathrm{~mm}$ throughout the experiment.

\section{Improvements}

After collecting the data presented herein, some improvements were made upon the apparatus in an attempt to remedy certain issues. These are shown in Figure 6.

During experimentation, inconsistent amplitude measurements were observed, and the accuracy of our methodology for obtaining these measurements fell under scrutiny. In an attempt to remedy these inconsistencies, a more detailed method was adopted. Rather than obtaining a single measurement of the upper portion of the waveform, multiple measurements were taken for the upper and lower halves and averaged, resulting in two amplitudes. A second average was then taken between these two values for a final result. Unfortunately, this did not significantly reduce the measurement error. It was decided that direct measurements of acceleration could be obtained digitally using accelerometers. By placing one of these sensors on each of the four corners of the shaker head, the phase of motion could also 
be compared and imbalances investigated. The analog accelerometer output was read by the Arduino UNO programmable microcontroller and converted to a Io-bit representation (a number between $\mathrm{O}$ and I023). The Arduino then sent this information to the serial stream, where it was retrieved by a Matlab program written to convert it into acceleration units and perform some basic analysis.

A second improvement upon the apparatus came in the form of thermal insulation placed between the shaker head and the dish containing the silicon liquid. During operation, eddy currents would cause the aluminum load block to heat up. In the higher ranges of frequency and amplitude, this effect was strong enough to heat the block to scalding temperatures. It was observed that the additional heat energy was causing patterns to form at a lower threshold, which badly skewed the results obtained. Until a more suitable material could be found to use for this purpose, a flat piece of wood with uniform thickness kept wet with water sufficed. It is expected that ceramic would provide adequate temperature isolation without deforming in response to changes in acceleration.

\section{Results}

For silicon fluids having kinematic viscosities of IO, 20, and $38 \mathrm{cSt}$, we systematically increased the amplitude and frequency of the driving waveform. Once patterns formed, these values were recorded and used to calculate the maximum acceleration achieved by the sample according to $\mathrm{x}=\mathrm{A}(2 \pi f)^{2}$ where A denotes amplitude, $f$ frequency and $\mathrm{x}$ acceleration in SI units. The acceleration was then represented in terms of Earth's gravitational acceleration, g. The resulting threshold values are shown in Figure 7. Interestingly, there appears to be no significant dependence of pattern formation threshold upon the viscosity of the liquid for Io and $20 \mathrm{cSt}$ viscosities. This is in stark opposition to the results found in a similar study by A. Kudrolli and J.P. Gollub (1996), which showed that formation thresholds are heavily dependent upon viscosity. This discrepancy could be due to the fact that the data for each viscosity was taken in an environment that was not temperature controlled. Since each data set was taken during different seasons of the year, the temperature of the liquid may have differed by as much as I5 degrees Fahrenheit. As was previously mentioned, higher temperatures cause formation thresholds to occur at lower accelerations, skewing results. The $38 \mathrm{cSt}$ formation threshold, on the other hand, occurs at a greater acceleration overall than the Io and $20 \mathrm{cSt}$ cases. This is the behavior expected. Since deformation of a more viscous liquid's surface requires more energy, it would follow that patterns in a more viscous liquid require a greater acceleration to form. These results offer some insight into how viscosity affects formation threshold, but to obtain a generalized trend, data for other viscosities must be obtained.

Pattern size was observed to decrease for increases in frequency, as seen by comparing the sizes of the patterns pictured in Figure 3, taken at $36 \mathrm{~Hz}$, and Figure 4, taken at $24 \mathrm{~Hz}$. This relationship was quantified using the k-vector magnitude, called the wavenumber, which is inversely proportional to the wavelength. In the context of the patterns studied herein, wavelength can be found by measuring the separation between peaks. The wavenumber is thus proportional to the inverseof the peak separation. This value was obtained for the patterns formed at the investigated frequencies and accelerations. The relationship between wavenumber and frequency, shown in Figure 8, was found to be inversely linear, as was expected from visual observation. This relationship shows us how the silicon liquid responds 
to the forcing frequency. Its linear nature means that the frequency of the waves formed is directly related to the frequency of the forcing.

\section{Conclusions}

In this experiment, silicon liquids having kinematic viscosities of Io, 20, and $38 \mathrm{cSt}$ were oscillated in the vertical direction. It was found that the plots of threshold accelerations for the io and $20 \mathrm{cSt}$ liquids were similar in shape and value, whereas the plot for the $38 \mathrm{cSt}$ liquid differed in both ways. The result for IO and $20 \mathrm{cSt}$ viscosities opposes the findings of Kudrolli and Gollub by suggesting that, in some cases, the formation threshold does not depend upon viscosity. Further investigation of this system is expected, mainly to obtain threshold data for at least two more viscosities. It was also found that the relationship between peak separation and frequency is inversely linear. This was expected due to previous qualitative observation.

It is expected that future investigations will focus on the phenomenon of stochastic resonance, where noise added to the signal affects formation thresholds. It is also expected that the addition of purely random, non-periodic noise would lower the amplitude of formation. Additional future plans involve applying a complex waveform to the shaker, specifically the superposition of two sine waves with systematically applied differences in phase, wavelength, and amplitude. Other studies have shown that this regime provides for a richer variety of patterns than were found herein. Once this system is sufficiently well-understood, the test sample will graduate to something granular, such as sand, cornstarch, or steel shot. 


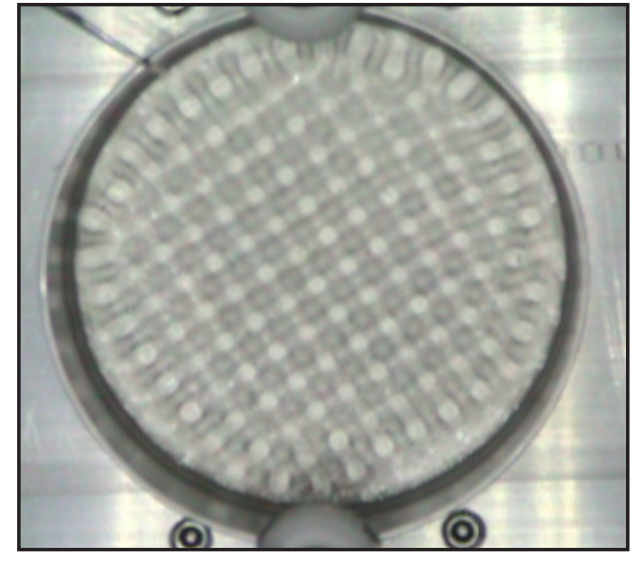

Figure I. $20 \mathrm{St}, 52 \mathrm{~Hz}$. Pure standing squares. This shape is created by two sets of waves crossing at $90^{\circ}$ of each other.

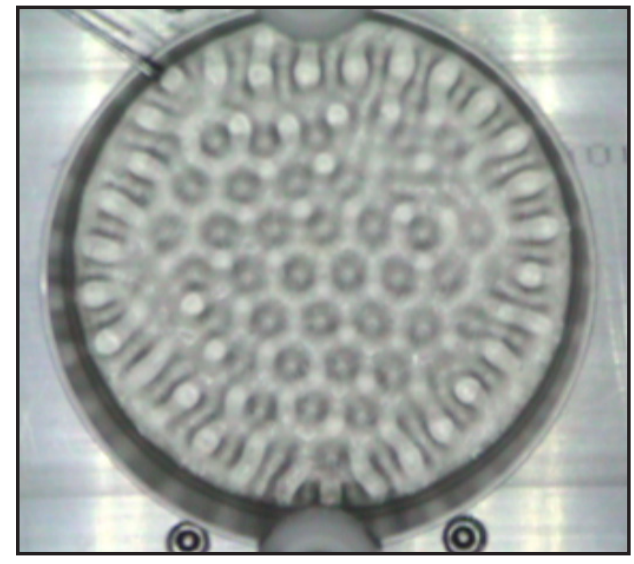

Figure 2. IocSt, $44 \mathrm{~Hz}$. Pure standing hexagons. This shape is formed when three waves cross at $120^{\circ}$ of each other.

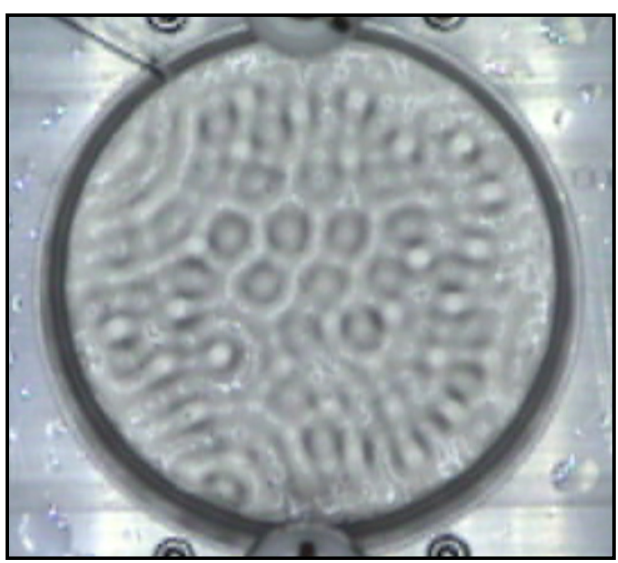

Figure 3. $20 \mathrm{cSt}, 36 \mathrm{~Hz}$. A screenshot of the chaotic, time-dependent combination of lines, squares, and hexagons.

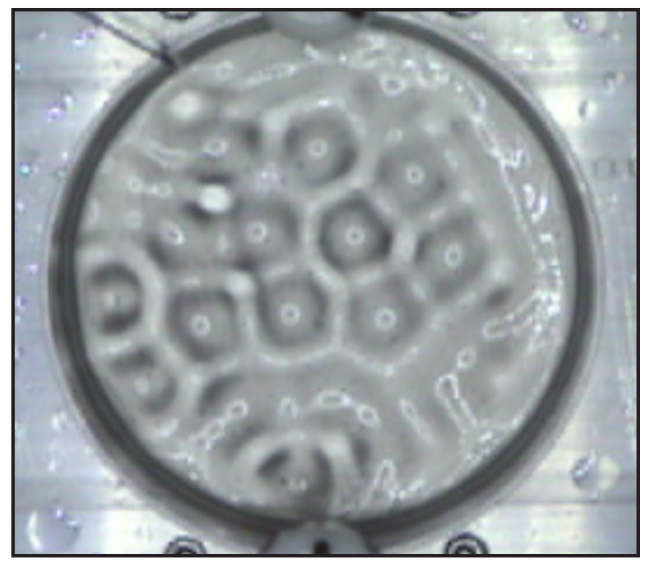

Figure 4. $20 \mathrm{SSt}, 24 \mathrm{~Hz}$. A second instance of the chaotic, time-dependent combination under different circumstances. 


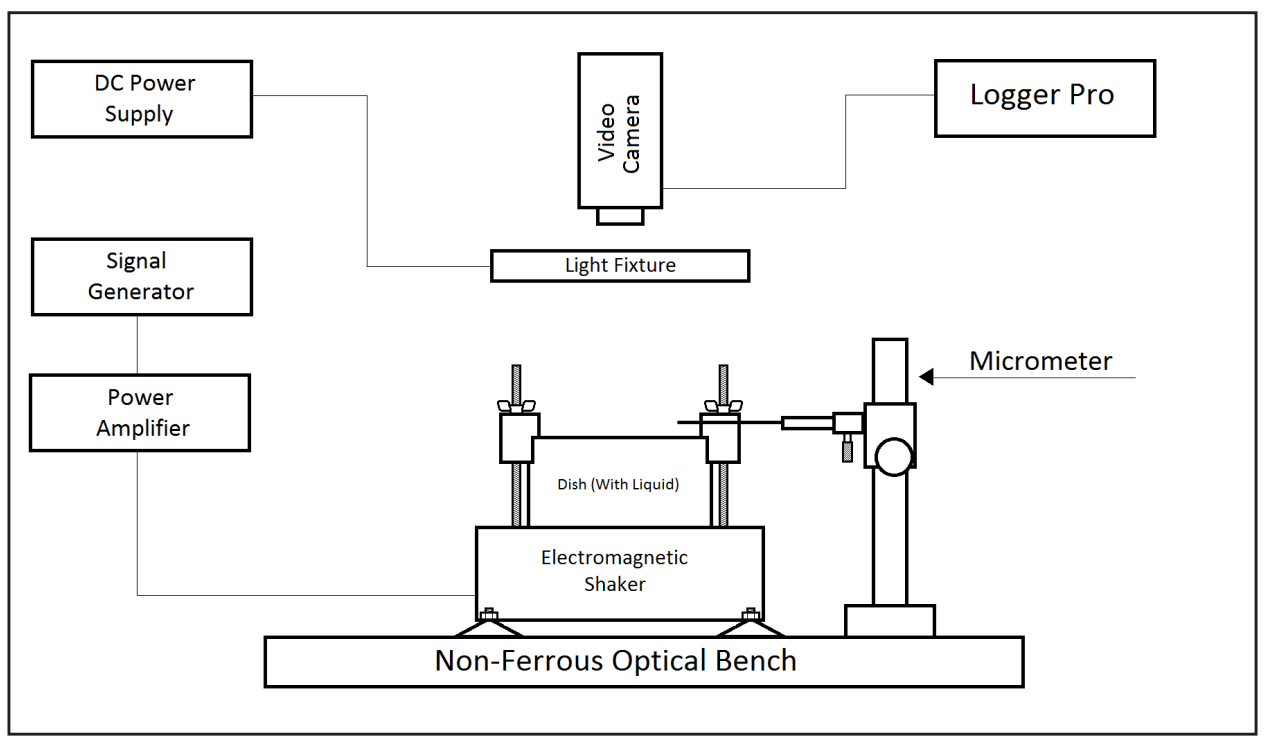

Figure 5. Diagram of experimental apparatus used to obtain data.

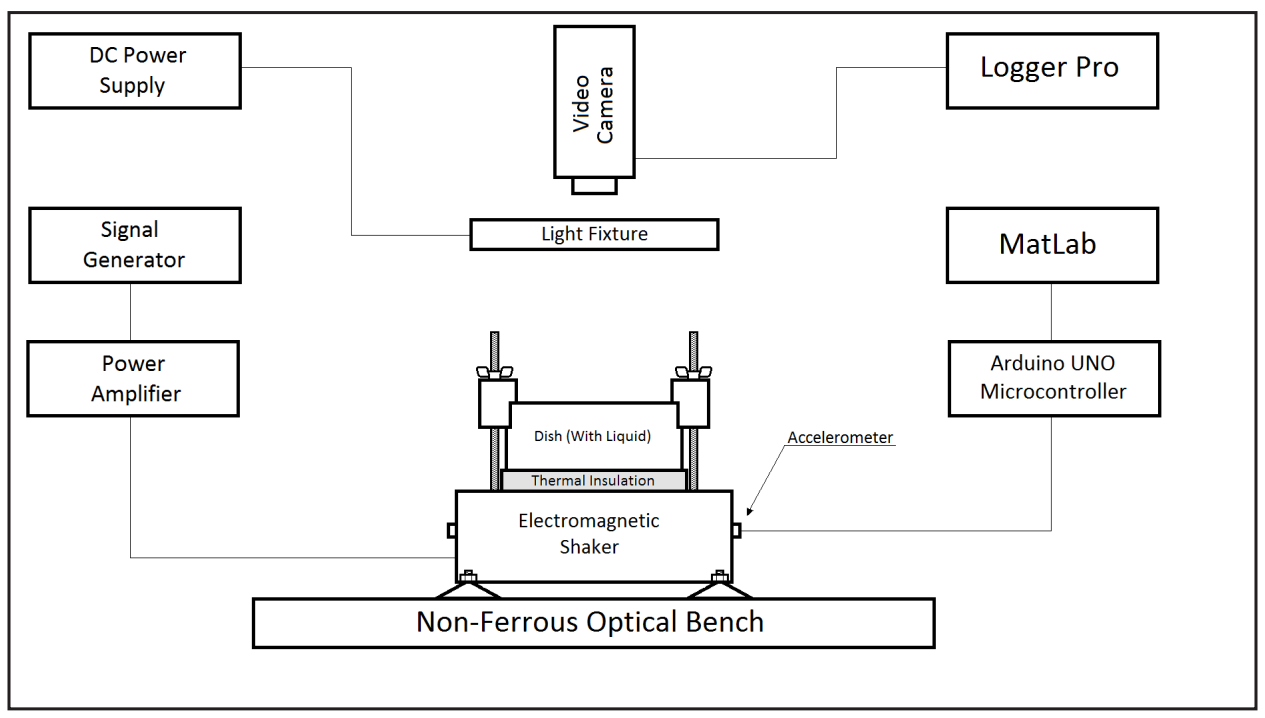

Figure 6. Experimental apparatus, with improvements. Thermal insulation was added between the shaker and sample as well as the accelerometers and their associated data acquisition system. 


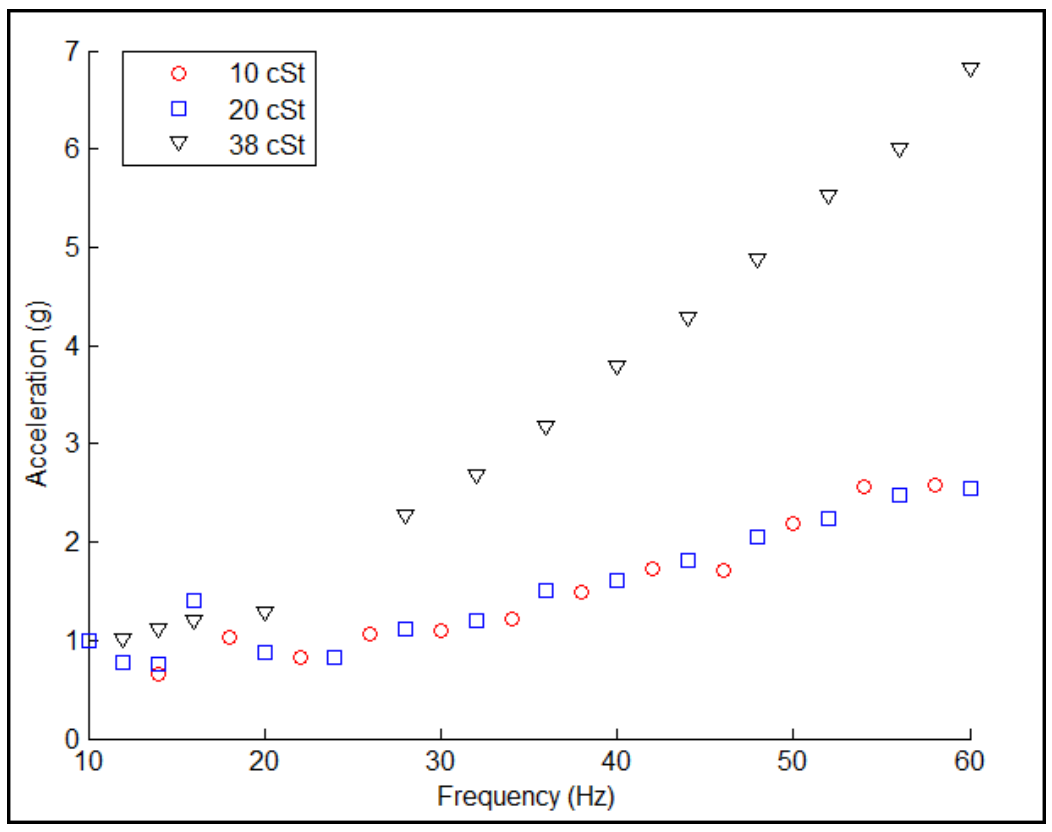

Figure 7. Pattern formation thresholds for 10, 20, and $38 \mathrm{cSt}$ as a function of forcing frequency and acceleration. Note that the thresholds of 10 and $20 \mathrm{cSt}$ are similar.

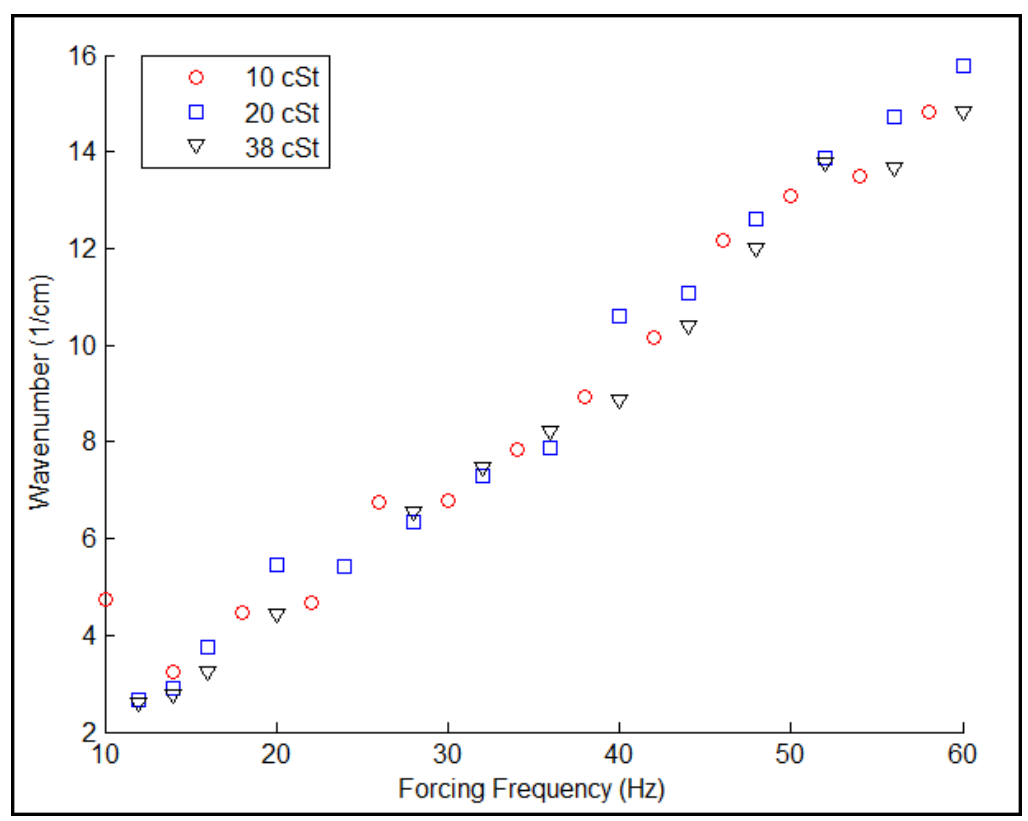

Figure 8. The relationship between pattern wavenumber and forcing frequency. As was expected from experimental observation and theory, this relationship is linear. 


\section{References}

Benjamin, T.B. \& Ursell, F. (1954). "The stability of the plane free surface of a liquid in vertical periodic motion." Proceedings of the Royal Society of London, 225, 505-515.

Kudrolli, A. \& Gollub, J.P. (1996). "Patterns and spatiotemporal chaos in parametrically forced surface waves: a systematic survey at large aspect ratio." Physica D, 97,I33-I54. 


\section{ABOUT THE AUTHOR}

Lisa M. Slaughter, a Cal Poly alumna, has a physics degree with minors in mathematics and music. She aspires to become an experimental physicist and obtain her PhD so her colleagues will call her "Dr. Slaughter." She finds physics interesting because the elegance of the universe never ceases to amaze her. "It is a privilege and a gift to have such tools as mathematics and the scientific method with which to probe its inner workings," she said. Lisa's research article submission was inspired by the mentorship she received from Dr. Nilgun Sungar. She believes that the Cal Poly Physics department exhibits the "Learn By Doing" philosophy by interweaving a core physics education with programming, computation and electronics. Because of these skills, Lisa has earned many employment opportunities and has become a more competitive and confident job applicant. "The intense lab experiences and impassioned discussions I had at Cal Poly helped me determine where I belong in the working world and what I need in order to be happy in my employment," she said. 